\title{
Investigation of novel biomarkers of drug-induced kidney injury in renal transplant recipients undergoing graft biopsy
}

Arthur Michon ( $\nabla$ art.michon@gmail.com )

Assistance Publique - Hôpitaux de Paris https://orcid.org/0000-0002-8678-3733

Antoine Durrbach

Hopital Bicetre

Jean-Charles Gautier

Sanofi

Xavier Benain

Sanofi

Catherine Lunven

Sanofi

Alexandre Jagerschmidt

Sanofi

Catherine Aubert

Sanofi

Oliver Poetz

Eberhard Karls Universitat Tubingen

Thomas Joos

Eberhard Karls Universitat Tubingen

Thierry Gury

Sanofi

Laurent Becquemont

Hopital Bicetre

Research article

Keywords: DIKI, biomarkers, renal transplantation, renal graft injury, renal graft biopsy

Posted Date: August 27th, 2019

DOI: https://doi.org/10.21203/rs.2.13591/v1 
License: (c) (i) This work is licensed under a Creative Commons Attribution 4.0 International License. Read Full License 


\section{Abstract}

Urinary and blood kidney biomarkers (BM) remain insufficient to detect early kidney injury. Our aim was to compare new kidney BM with histopathological data in kidney allograft recipients. Blood and urine samples of consecutive adult patients were collected just prior graft biopsy. All kidney samples were classified according to Banff 2007. The diagnostic performance (area under ROC curve, AUROC) of 16 new BM was compared to those of urinary proteins, blood urea nitrogen, eGFR and serum creatinine to identify histopathological groups. 223 patients were analyzed. Versus slightly modified renal parenchyma (SMRP), microalbuminuria and urinary proteins had the highest diagnostic performance toward glomerular injury. Urinary neutrophil gelatinase associated lipocalin (NGAL) had the best performance values for acute tubular necrosis (ATN) versus SMRP (AUROC 0.93). Others BM reached slightly lower AUROC reaching 0.89 . When comparing ATN to acute rejection several new urinary BM (NGAL, cystatin C, and MCP1) and classical BM (eGFR, serum creatinine) reached similar AUROC values ranging from 0.80 to 0.85 . Values of urinary NGAL were 10 -fold higher in ATN compared to acute rejection $(p=0.0004)$. New BM did not outperform classical BM during renal transplantation. Urinary NGAL might be helpful to discriminate ATN and acute rejection.

\section{Background}

Kidney injury, which occurs in many diseases, is poorly identified because of the absence of early markers and is mostly identified in case of severe renal lesions with impaired renal function. Urinary or blood BM would be helpful for the diagnosis of initial kidney injury and early intervention. In Nephrology, the most used BM are serum creatinine and blood urea nitrogen (BUN), as well as proteinuria or blood cells in urine. However, these parameters have a poor sensitivity, are responsible for a diagnostic and/or therapeutic delay and a poor specificity frequently requiring the use of a renal biopsy for an adequate characterization of injuries. Unfortunately, renal biopsy is an invasive procedure exposing patients to rare but sometimes life-threatening hemorrhagic adverse events (1).

During the last decade, efforts have been made to discover and characterize new early diagnostic urinary BM (2)(3)(4)(5)(6). Simultaneously, the FDA and EMA have also encouraged research programs to identify and qualify early BM of drug induced kidney injury (DIKI) (7). These new renal BM (Figure 1) can be classified as functional BM or tissue injury BM.

Microalbuminuria is a BM of glomerular injury and/or impaired tubular proximal protein reabsorption. Cystatin $\mathrm{C}$ is a low-molecular weight protein freely filtered in the glomerulus and then reabsorbed by renal proximal tubules. In addition to its potential role as a blood biomarker of glomerular filtration rate, urinary cystatin $C$ is a marker of impaired proximal tubule function. Retinol-binding protein-4 (RBP4), a protein of the lipocalin family is a BM of impaired proximal tubule reabsorption. Nephrin and podocin (components of the filtration splits of podocytes), Kirrel 1 (a protein that interacts with the $\mathrm{C}$ terminus of podocin) and podocalyxin (a major constituent of the glycocalyx of podocytes) are new BM of glomerular injury. Nacetyl- $\beta$-D-glucosaminidase (NAG) (a brush border enzyme in proximal renal tubular cells), neutrophil 
gelatinase associated lipocalin (NGAL) (a protein expressed in neutrophils and various tissues at low levels, but induced in epithelial cells during inflammation) (8), Kidney injury molecule-1 (KIM1) (a transmembrane protein whose expression is increased in case of proximal tubular injury and/or regeneration) and clusterin (a protein associated with the clearance of cellular debris and apoptosis) are promising BM of proximal tubular injury. Among these proximal tubular cell biomarkers, NGAL is a highly attractive biomarker for the sensitive monitoring of DIKI in clinical trials because it is expressed at low levels under normal conditions, but rapidly upregulated following kidney injury (9). Osteopontin, a secreted glycoprotein and calbindin, a renal calcium-binding protein are used as markers of distal tubular injury. Aquaporin 2 allows water reabsorption and the increase of its urinary level is a marker of collecting duct injury. Monocyte chemotactic protein-1 (MCP1) is a marker of inflammation that recruits leucocytes to the sites of inflammation. Most of these new renal BM still remain under investigation in humans and have not been validated as clinically useful BM in routine practice $(10)(11)(12)(13)(14)$, although NGAL seems the most promising (15)(16). The links between histological lesions, the current nephrology gold standard, and blood or urinary levels of these new BM remains to be established in the clinical setting.

In renal graft recipients, renal biopsies are performed as a routine evaluation mainly in the first semester after transplantation. Renal dysfunction is frequent after transplantation and may be linked to drug toxicity, immunologic injury of humoral or cellular origin, infectious injury, or recurrence of the initial disease for which no current BM are available to discriminate the type and the consequence of injury. Therefore, biopsies are routinely performed to identify initial lesions. These graft biopsies performed in every graft recipient represent an ideal opportunity to evaluate new renal BM and assess their association with renal histopathology, the current nephrologic gold standard.

Therefore, the aim of this exploratory study was to assess diagnostic accuracy of 16 new urinary and blood renal BM reflecting injury in different segments of the nephron and compare to renal histopathological data.

\section{Methods}

\section{Patients}

Male or female renal transplant recipients, aged 18 or over, who qualified for a renal biopsy as a routine evaluation at 3 month or because of an impaired renal function, and for whom blood and urine collection was possible, were considered for eligibility. Patients could not be included if they received nephrotoxic drugs other than those administered as standard care for renal transplant, within 1 month prior to screening until the last sample collection. Drugs known to alter tubular secretion of creatinine (e.g., cimetidine, amiodarone, dronedarone) but not cotrimoxazole, taken within 7 days prior to screening until the last sample collection, were additional exclusion criteria.

Inclusion started on July the $6^{\text {th }} 2011$ and ended on March the $11^{\text {th }} 2015$.

\section{Methodology}


This prospective, single-center, open label study was conducted in the nephrology department of Bicêtre University Hospital, APHP. It was part of the European Innovative Medicines Initiative Joint Undertaking (IMI JU) Safer and Faster Evidence based Translation (SAFE-T) Consortium aimed at the clinical biomarker qualification for drug-induced injury to kidney, liver and the vascular system. All the drugs and their doses were left to the treating nephrologists, using "real world" treatment options.

All the patients gave their written informed consent to participate.

The study was approved by the French drug agency (ANSM) the 22 September 2010 (ID RCB : 2010A01050-39) and a French ethical research committee (CPP lle de France VII, ID : 11-004).

The duration of participation per patient (from screening to end-of-study) was 1 or 2 days depending on the time interval between blood and urine collection (Day 1), and the graft biopsy (Day 1 or Day 2).

Blood and urine samples were collected just prior graft biopsy.

Fasting blood samples were collected for plasma (on Li-Heparin and EDTA) and serum preparation (in a dry tube). The blood samples were centrifuged at $+4^{\circ} \mathrm{C}$ at $2600 \mathrm{~g}$ for $10 \mathrm{~min}$, within $30 \mathrm{~min}$ of collection for plasma samples, and after $30-60$ min of clotting at $+37^{\circ} \mathrm{C}$ further 30 min at $+4^{\circ} \mathrm{C}$ for serum sample. Immediately following centrifugation, 3 aliquots $(0.5 \mathrm{~mL}$ each) of the supernatant were transferred into 3 labeled polypropylene cryovials and immediately frozen at $-80^{\circ} \mathrm{C}$.

Fasting urine sample (spot urine) was collected in a dry container, after that he/she had emptied his/her bladder (overnight urine discarded) and immediately placed in an ice bath, then centrifuged at $+4^{\circ} \mathrm{C}$ at $3000 \mathrm{~g}$ for $5 \mathrm{~min}$, within $30 \mathrm{~min}$ of collection. Aliquots ( $1 \mathrm{~mL}$ each) of the supernatant were transferred into 12 labeled polypropylene cryovials, and they were immediately frozen at $-80^{\circ} \mathrm{C}$.

\section{Study endpoints}

a) Histological study endpoints:

All kidney samples were reviewed by the same trained pathologist who classified the renal biopsies according to Banff 2007 (17)(18) classification using the quantitative criteria for glomerulitis (" $\mathrm{g}$ "), tubulitis (" $t$ "), intimal arteritis (" $v$ "), interstitial infiltrate of mononuclear cell ("i"), and interstitial fibrosis ("ci").

The same pathologist also classified patients in 11 groups based on his histological conclusion.

b) Biomarker assays

Classical BM, serum creatinine, BUN, and urinary total proteins were measured as routine parameters at Bicêtre Hospital, eGFR was calculated according to MDRD modified formula (19). 
The novel urinary BM cystatin C, NGAL, clusterin, calbindin, KIM-1, osteopontin, and MCP1 were quantified by Natural Medical Sciences Institute (NMI) using multiplex Luminex immunoassays. Urinary NAG was measured at Sanofi with an enzymatic assay, urinary microalbumin and plasma cystatin $C$ with immunoturbidimetric assays. Urinary RBP4 was measured by NMI using immunoprecipitation LC/MS assay as previously described (20)(21) with amendment (see Additional file 1). Urinary aquaporin 2, podocin, podocalyxin, nephrin, and kirrel-1 were quantified at Sanofi by using an HPLC-MRM-MS multiplex method derived from previously developed singleplex LC/MS methods for aquaporin-2 (22), podocin (23) and podocalyxin (24) (see Additional file 1).

\section{Statistical methods (see Additional file 2)}

Descriptive statistics were presented as mean \pm SD, median \pm interquartile range.

All serum or blood renal BM were analyzed as raw data. All urinary renal biomarkers were analyzed as raw data corrected by urinary creatinine.

Empirical ROC curves were constructed for each BM using patients belonging to a clinical group (e.g. ATN, GP, ACR...) as patients belonging to the event group versus patients belonging to the SMRP (or all the other patients) as patients belonging to the non-event group; if the number of patients in the event group was lower or equal than 5 then no ROC curves were constructed.

For all cases, combination of biomarkers (after log transformation of BM values, except for eGFR) were explored by selection of the best BM combination (in terms of lowest Akaike criteria) containing 3 BM. AUROC bootstrap estimates and $95 \% \mathrm{Cls}$ were provided for all ROC curves of each individual BM and combination of BM.

For individual and combination of BM (when AUROC $>0.7$ ), bootstrap best BM's diagnostic threshold and associated $95 \% \mathrm{Cls}$ were estimated by looking for the pair of estimated sensitivity/specificity closest to perfect sensitivity and specificity. Sensitivity and specificity bootstrap estimates and $95 \%$ Cls associated with this best BM's diagnostic threshold were also calculated.

Estimates were calculated using bootstrap methods in order to lighten the overfitting issue related to the search of the best biomarker among several ones.

\section{Results}

\section{Patients}

Two hundred fifty-seven patients were included in this study from the nephrology department of Bicêtre University Hospital. Twenty-three included patients did not undergo a graft biopsy because of urinary infection or hemostatic abnormalities and were not retained in the present analysis, as well as 11 additional patients for whom no analyzable kidney tissue could be obtained. Thus, 223 patients were 
retained for the present BM study. Patient characteristics at baseline for BM population are presented in Table 1.

Patients were classified in 11 groups based on renal histology. Two patients could not be classified due to inadequate renal parenchyma (Table 2).

Only groups containing strictly more than 5 patients were considered for further statistical analysis. Thus 5 groups were not included in this analysis due to their small numbers of patients, except when comparing to "all other patients".

Graft biopsies were also classified according to BANFF 2007 for the 5 main lesion types, for which severity was graded from 0 to 3 (Table 3 ).

\section{Descriptive statistics for biomarkers}

The range levels of BM normalized to urinary creatinine for all patients are presented in Table 4.

The study of covariates (age and gender) which might affect blood or urinary BM levels was performed to avoid hypothetical bias. No statistically significant $(p<0.05)$ impact was observed for age on any BM, except for serum creatinine (female versus male geometric mean ratio of 0.82 [95\% Cl: 0.68, 0.99; $p$-value $=0.0393]$ ).

No relevant covariate impact was observed on any covariate adjusted ROC analysis.

३BM performance to detect the severity inside each histological class

When looking at the severity of each anatomical lesion, only total urinary proteins, microalbuminuria and NGAL had the potential to distinguish (AUROC value above 0.8 ) from one grade to another inside each anatomical class (Additional file 3). Urinary NGAL seemed to be of interest to distinguish severe (ci3) from the other grades (ci0 to ci2) of interstitial fibrosis (AUROC 0.81).

\section{BM performance for main histopathological diagnosis}

Each main histopathological diagnostic group containing more than 5 patients was compared toSMRP as control group. Table 5 presents the ranking of BM by AUROC. The same analysis was performed using all the other study patients as control group instead of SMRP group.

Briefly, total urinary proteins and microalbuminuria had equal performance for the diagnosis of glomerulopathy regardless of the control group (SMRP or all the other patients). NGAL was the best BM for the diagnosis of ATN reaching an AUROC of 0.93 with a $91 \%$ sensitivity and specificity. Other new BM such as serum cystatin $\mathrm{C}$ and urinary cystatin $\mathrm{C}, \mathrm{RBP} 4, \mathrm{KIM} 1$ and clusterin reached similar AUROC ranging from 0.84 to 0.89 , like the classical $B M$ serum creatinine, BUN and urinary total proteins (AUROC of $0.87,0.88$ and 0.89 respectively). 
BUN was the best BM for AHR diagnosis with an AUROC of 0.82 , although its sensitivity and specificity remained below $85 \%$. An association of BM might be of interest for AHR diagnosis. For example, the association of BUN, NGAL and microalbuminuria reached an AUROC of 0.91 , with $89 \%$ sensitivity and $88 \%$ specificity (Additional file 4). These analyses of combinations of biomarkers are for exploratory purpose only.

No single BM or combination of BM had relevant performance for the diagnosis of ACR or IFTA.

\section{BM performance to discriminate ATN from graft rejection during early transplantation}

During the first days of renal transplantation whereas renal function progressively improves, a recurring question arises concerning the possibility of an underlying graft rejection (ACR or AHR) or the normal recovery from an ATN. In order to assess the potential utility of these new BM to discriminate the 2 diagnosis (that might avoid a graft biopsy) we tested their diagnostic performance by comparing ATN to $A C R, A H R$ or ACR+AHR. The only patient with mixed humoral and cellular rejection (AMR) was included in ACR and AHR groups.

When comparing ATN to ACR, the new BM did not improve the diagnostic performance of classical BM (Table 6). However, an association of BM including NGAL might increase the diagnostic performance with $90 \%$ sensitivity and $95 \%$ specificity (Additional file 5). These analyses of combinations of biomarkers are for exploratory purpose only.

When comparing ATN to AHR, the new BM did not improve the diagnostic performance of classical BM (Table 6). Once again, an association of BM including NGAL might increase the performance values (Additional file 5).

Comparison of ATN versus both ACR and AHR showed that AUROC values were similar for several new urinary BM (NGAL, cystatin C, and MCP1) and the classical BM eGFR and serum creatinine. Again, the association of several BM including NGAL seemed to increase the performance values (Additional file 5) reaching $91 \%$ sensitivity and $93 \%$ specificity, even if the very few number of patients makes these results exploratory only.

In this specific context of early renal transplantation, it is of note the values of urinary NGAL which discriminate ATN from rejection were about 10 -fold higher in patients with ATN (geometric mean value of $64.0 \mu \mathrm{g} / \mathrm{mmol}$ ) compared to patients with ACR (geometric mean value of $5.6 \mu \mathrm{g} / \mathrm{mmol}, \mathrm{p}=0.0009$ ) or AHR (geometric mean value of $7.4 \mu \mathrm{g} / \mathrm{mmol}, \mathrm{p}=0.0142$ ), or acute rejection (AHR + AMR, geometric mean value of $10.7 \mu \mathrm{g} / \mathrm{mmol}, p=0.0004)$. The best threshold levels to discriminate ATN from ACR, AHR and acute rejection (both ACR and AHR) were $12.0 \mu \mathrm{g} / \mathrm{mmol}$, and $45.2 \mu \mathrm{g} / \mathrm{mmol}$ and $17.7 \mu \mathrm{g} / \mathrm{mmol}$, respectively.

\section{Discussion}


This study aimed to assess diagnostic accuracy of selected urinary and blood renal BM reflecting lesions in different segments of the nephron compared to renal histopathological data. Our results revealed that when taken individually the new BM did not outperform the classical BM currently used in clinical practice. However, urinary NGAL seems to be potentially helpful to differentiate graft rejection from ATN recovery during early renal transplantation.

\section{1 - BM association with each BANFF anatomical structure lesion:}

When comparing the BM by histological lesion types and severity, total urinary proteins and microalbuminuria were the most performant BM to detect and quantify glomerular injury, as expected (25)(26). NGAL could only differentiate severe interstitial fibrosis (ci3) from ci0 to ci2, in agreement with Nickolas et al. (27) who observed an association between NGAL and interstitial fibrosis among nongrafted CKD patients. No other single BM was shown to be associated to any other lesion of the BANFF anatomical structures.

\section{2 - BM association with the main histopathological diagnosis}

In our study, NGAL had the best performance values to diagnose ATN versus SMRP. Other new BM such as cystatin C, RBP4, KIM1 and clusterin were identified as potential BM reflecting ATN, but their performances were slightly lower than NGAL, and similar to those of classical BM.

Urinary neutrophil gelatinase associated lipocalin (NGAL) had the best performance values for acute tubular necrosis (ATN) versus SMRP (AUROC $0.93(95 \% \mathrm{Cl} 0.85-0.99)$ ). Other new BM (serum or urinary cystatin-C, RBP4, KIM1 and clusterin) and classical BM reached slightly lower AUROC ranging from 0.84 to 0.89 .

NGAL has been the most studied, particularly for AKI during cardiac surgery and critical illness (9).

In renal transplantation, NGAL has been particularly studied to predict the risk of delayed graft function (28) as a more efficient BM than serum creatinine (29). Since delayed graft function is mainly consecutive to ATN, our results are in agreement with previous data (NGAL a good candidate BM for ATN). Kaufeld et al. (30) showed that NGAL levels were 2-fold higher in transplant patients who developed biopsy-proven ATN compared to those free from ATN, with a significant overlap between both groups. Recently, Moledina et al. (31) found that NGAL's performance was better than that of serum creatinine in distinguishing deceased donors with or without ATN.

In the present study, KIM1 was also identified as a candidate BM for the diagnosis of ATN, but it performed less well than NGAL or urinary cystatin C. Such results are in line with those from Yadav et al. (32) who showed that urinary KIM1 can predict delayed graft dysfunction among biopsy-proven ATN patients.

3 - Evaluation of BM to differentiate ATN from graft rejection 
Among the new BM of kidney injury, urinary NGAL and cystatin C may potentially help to distinguish between patients with ATN and graft rejection. Serum creatinine and eGFR seemed to do so as well. The performance values of urinary NGAL increased when combined with some others BM, like KIM1 and podocalyxin, but these combinations have been evaluated for exploratory purpose only, to be further evaluated with higher number of patients.

Kohei et al. (33) showed that NGAL in both serum and urine is a powerful BM of acute rejection during the early period after living-donor kidney transplantation, but the authors did not compare the patients with a rejection to patients with an ATN. Other authors evaluated NGAL to discriminate AKI from acute rejection in renal allograft recipients and obtained less impressive diagnostic performance (34)(35)(36)(37), but much later after the transplantation or without histological controls or prerenal AKI as comparators.

The ability of NGAL to differentiate rejection from other causes of AKI is still debated. Our results suggest that a threshold level of NGAL between 15 and $20 \mu \mathrm{g} / \mathrm{mmol}$ (17.7 in our study) might help to discriminate ATN from acute rejection during early transplantation. Moreover, the combination of different BM could increase its performance values.

\section{Limitations}

Our study has several limitations. The main one is the heterogeneity of the study population with several causes of renal injuries. Moreover, most of the patients had multiple types of kidney injuries (tubular, interstitial, glomerular and vascular). Therefore, it was difficult to distinguish between the influence of the main histological lesion on BM levels, and the effect of the associated lesions. Another limitation is the relatively small number of patients included in each histopathological diagnostic group; consequently, the precision (IC95\%) of the reported sensitivities and specificities was for some groups relatively weak. Our results need to be replicated in independent groups of renal allograft recipients. Finally, it is important to keep in mind that these selected BM were developed for the particular situation of drug induced kidney injuries (DIKI), which only concerned a small part of our study population.

\section{Strength}

The main strength of this study is the availability of concomitant renal histological data and BM concentration for every patient. Its prospective design among consecutive patients limits the bias as well as the constant quality of the samples. Finally, we compared classical BM to new BM that allows the evaluation of new BM in the context of standard care.

\section{Conclusions}

Newly studied BM did not seem to have better performance than classical BM to identify the type or the severity of kidney injury during renal transplantation, except NGAL which might have slightly better performance values than the other BM. During the initial phase of renal transplantation, when hesitating between ATN recovery and acute graft rejection, urinary NGAL might be helpful to discriminate between 
these 2 diagnoses. This is a common situation in clinical practice, and there is a real need of early noninvasive diagnostic tools, to potentially avoid some graft biopsies and to reduce diagnosis delay. Future prospective studies may focus on new BM such as NGAL in this situation, to improve their use in clinical practice.

\section{Declarations}

Ethics approval and consent to participate:

The study was approved by the French drug agency (ANSM) the 22 September 2010 (ID RCB : 2010A01050-39) and a French ethical research committee (CPP lle de France VII, ID : 11-004).

All the patients gave their written informed consent to participate.

Consent for publication : Not applicable.

Availability of data and material: The dataset supporting the conclusions of this article is included within the additional file 6 .

Competing interests:

A.M. and L.B. : close family member working at Sanofi.

Others authors don't have any conflict of interest.

\section{Funding:}

The study was funded by Sanofi as part of its in-kind contribution to the SAFE-T Consortium. Oliver Poetz was supported by the German Federal Ministry of Education and Research grant FKZ 031B0395 (GO-Bio).

\section{Authors' contributions}

A.M. and A.J. contributed in writing the article.

A.D. and L.B. contributed in research design, conduct of the study and writing the article.

J.-C.G. contributed in research design and writing the article.

X.B. contributed in research design, statistical analysis and writing the article.

C.L. contributed in research design, setting and conduct of the study.

C.A., O.P., T.J., T.G. contributed in biomarker data acquisition. 
The authors would like to thank colleagues from the European IMI-SAFE-T consortium for helpful discussions. We are grateful to Isabelle Clavier, Olivier Pasquier, Sylvie Brohier, Pascal Le Priol and Nathalie Laufer from Sanofi for their participation in this study

\section{Abbreviations}

ACR, acute cellular rejection

AHR, acute humoral rejection

AKI, acute kidney injury

AMR, acute mixed rejection

ATN, acute tubular necrosis

AUROC, area under ROC curve

BKVN, BK virus nephropathy

$\mathrm{BM}$, biomarkers

BUN, blood urea nitrogen

$\mathrm{CCR}$, chronic cellular rejection

CIT, calcineurin inhibitor toxicity

DIKI, drug-induced kidney injury

GP, glomerulopathy

IFTA, interstitial fibrosis and tubular atrophy

KIM1, kidney injury molecule-1

MCP1, monocyte chemotactic protein-1

NAG, N-acetyl- $\beta$-D-glucosaminidase

NGAL, neutrophil gelatinase associated lipocalin 2

RBP4, retinol-binding protein-4

SMRP, slightly modified renal parenchyma

TMA, thrombotic microangiopathy 


\section{References}

1. Prakash J, Singh M, Tripathi K, Rai US. Complications of percutaneous renal biopsy. J Indian Med Assoc. déc 1994;92(12):395-6.

2. Sundaram R, Abhirama B, Gowtham L, Kalpana G, Sudha M, Thiyagarajan T, et al. Limitations of Conventional Parameters and Role of Urinary Protein Biomarkers in the Determination of Drug Induced Acute Kidney Injury in Very Early Stage. Br J Pharm Res. 10 janv 2014;4(21):2463-74.

3. Mariat C. Diagnostic et suivi de la dysfonction chronique du greffon rénal: du DFG aux nouveaux biomarqueurs. Néphrologie Thérapeutique. oct 2008;4:S204-7.

4. Muthukumar T, Dadhania D, Ding R, Snopkowski C, Naqvi R, Lee JB, et al. Messenger RNA for FOXP3 in the Urine of Renal-Allograft Recipients. N Engl J Med. déc 2005;353(22):2342-51.

5. Ho J, Rush DN, Nickerson PW. Urinary biomarkers of renal transplant outcome: Curr Opin Organ Transplant. août 2015;20(4):476-81.

6. Hamdorf M, Kawakita S, Everly M. The Potential of MicroRNAs as Novel Biomarkers for Transplant Rejection. J Immunol Res. 2017;2017:1-12.

7. Brott D, Adler S, Arani R, Lovick S, Pinches M, Furlong S. Characterization of renal biomarkers for use in clinical trials: biomarker evaluation in healthy volunteers. Drug Des Devel Ther. févr 2014;227.

8. Cowland JB, Borregaard N. Molecular Characterization and Pattern of Tissue Expression of the Gene for Neutrophil Gelatinase-Associated Lipocalin from Humans. Genomics. oct 1997;45(1):17-23.

9. Haase-Fielitz A, Haase M, Devarajan P. Neutrophil gelatinase-associated lipocalin as a biomarker of acute kidney injury: a critical evaluation of current status. Ann Clin Biochem Int J Biochem Lab Med. 1 mai 2014;51(3):335-51.

10. Gueguen Y, Rouas C, Leblond FA. Les biomarqueurs d'atteinte rénale. Néphrologie Thérapeutique. juin 2012;8(3):146-55.

11. Bonventre JV, Vaidya VS, Schmouder R, Feig P, Dieterle F. Next-generation biomarkers for detecting kidney toxicity. Nat Biotechnol. mai 2010;28(5):436-40.

12. Vanmassenhove J, Vanholder R, Nagler E, Van Biesen W. Urinary and serum biomarkers for the diagnosis of acute kidney injury: an in-depth review of the literature*. Nephrol Dial Transplant. févr 2013;28(2):254-73.

13. Nguyen MT, Devarajan P. Biomarkers for the early detection of acute kidney injury. Pediatr Nephrol. déc 2008;23(12):2151-7.

14. Mårtensson J, Martling C-R, Bell M. Novel biomarkers of acute kidney injury and failure: clinical applicability. Br J Anaesth. déc 2012;109(6):843-50.

15. Ronco C, Legrand M, Goldstein SL, Hur M, Tran N, Howell EC, et al. Neutrophil Gelatinase-Associated Lipocalin: Ready for Routine Clinical Use? An International Perspective. Blood Purif. 2014;37(4):271-85.

16. Devarajan P. Neutrophil gelatinase-associated lipocalin (NGAL): A new marker of kidney disease. Scand J Clin Lab Invest. janv 2008;68(sup241):89-94. 
17. Solez K, Colvin RB, Racusen LC, Haas M, Sis B, Mengel M, et al. Banff 07 Classification of Renal Allograft Pathology: Updates and Future Directions. Am J Transplant. avr 2008;8(4):753-60.

18. Racusen LC, Solez K, Colvin RB, Bonsib SM, Castro MC, Cavallo T, et al. The Banff 97 working classification of renal allograft pathology. Kidney Int. févr 1999;55(2):713-23.

19. Levey AS, Bosch JP, Lewis JB, Greene T, Rogers N, Roth D. A more accurate method to estimate glomerular filtration rate from serum creatinine: a new prediction equation. Modification of Diet in Renal Disease Study Group. Ann Intern Med. 16 mars 1999;130(6):461-70.

20. Gautier J-C, Zhou X, Yang Y, Gury T, Qu Z, Palazzi X, et al. Evaluation of novel biomarkers of nephrotoxicity in Cynomolgus monkeys treated with gentamicin. Toxicol Appl Pharmacol. juill 2016;303:1-10.

21. Hoeppe S, Schreiber TD, Planatscher H, Zell A, Templin MF, Stoll D, et al. Targeting Peptide Termini, a Novel Immunoaffinity Approach to Reduce Complexity in Mass Spectrometric Protein Identification. Mol Cell Proteomics. févr 2011;10(2):M110.002857.

22. Jaffuel A, Lemoine J, Aubert C, Simon R, Léonard J-F, Gautier J-C, et al. Optimization of liquid chromatography-multiple reaction monitoring cubed mass spectrometry assay for protein quantification: Application to aquaporin-2 water channel in human urine. J Chromatogr A. août 2013;1301:122-30.

23. Simon R, Lemoine J, Fonbonne C, Jaffuel A, Léonard J-F, Gautier J-C, et al. Absolute quantification of podocin, a potential biomarker of glomerular injury in human urine, by liquid chromatographymultiple reaction monitoring cubed mass spectrometry. J Pharm Biomed Anal. juin 2014;94:84-91.

24. Biarc J, Simon R, Fonbonne C, Léonard J-F, Gautier J-C, Pasquier O, et al. Absolute quantification of podocalyxin, a potential biomarker of glomerular injury in human urine, by liquid chromatographymass spectrometry. J Chromatogr A. 5 juin 2015;1397:81-5.

25. Rytand DA. The significance of albuminuria; proteinuria. Calif Med. mars 1949;70(3):174-6.

26. Urizar RE, Cerda J, Godley K, Haenel C, Curtis G. The early diagnosis of glomerulopathy through the detection of subclinical proteinuria: microalbuminuria. N Y State J Med. août 1988;88(8):423-6.

27. Nickolas TL, Forster CS, Sise ME, Barasch N, Valle DS-D, Viltard M, et al. NGAL (Lcn2) monomer is associated with tubulointerstitial damage in chronic kidney disease. Kidney Int. sept 2012;82(6):718-22.

28. Ramirez-Sandoval JC, Herrington W, Morales-Buenrostro LE. Neutrophil gelatinase-associated lipocalin in kidney transplantation: A review. Transplant Rev. juill 2015;29(3):139-44.

29. Lacquaniti A, Caccamo C, Salis P, Chirico V, Buemi A, Cernaro V, et al. Delayed graft function and chronic allograft nephropathy: diagnostic and prognostic role of neutrophil gelatinase-associated lipocalin. Biomarkers. 18 mai 2016;21(4):371-8.

30. Kaufeld JK, Gwinner W, Scheffner I, Haller HG, Schiffer M. Urinary NGAL Ratio Is Not a Sensitive Biomarker for Monitoring Acute Tubular Injury in Kidney Transplant Patients: NGAL and ATI in Renal Transplant Patients. J Transplant. 2012;2012:1-8. 
31. Moledina DG, Hall IE, Thiessen-Philbrook H, Reese PP, Weng FL, Schröppel B, et al. Performance of Serum Creatinine and Kidney Injury Biomarkers for Diagnosing Histologic Acute Tubular Injury. Am J Kidney Dis. déc 2017;70(6):807-16.

32. Yadav B, Prasad N, Agrawal V, Jaiswal A, Agrawal V, Rai M, et al. Urinary Kidney injury molecule-1 can predict delayed graft function in living donor renal allograft recipients: Kidney injury molecule and DGF. Nephrology. nov 2015;20(11):801-6.

33. Kohei J, Ishida H, Kazunari T, Tsuchiya K, Nitta K. Neutrophil gelatinase-associated lipocalin is a sensitive biomarker for the early diagnosis of acute rejection after living-donor kidney transplantation. Int Urol Nephrol. août 2013;45(4):1159-67.

34. Heyne N, Kemmner S, Schneider C, Nadalin S, Königsrainer A, Häring H-U. Urinary Neutrophil Gelatinase-Associated Lipocalin Accurately Detects Acute Allograft Rejection Among Other Causes of Acute Kidney Injury in Renal Allograft Recipients: Transplant J. juin 2012;93(12):1252-7.

35. Ramirez-Sandoval JC, Barrera-Chimal J, Simancas PE, Correa-Rotter R, Bobadilla NA, MoralesBuenrostro LE. Tubular urinary biomarkers do not identify aetiology of acute kidney injury in kidney transplant recipients: AKI biomarkers in kidney transplantation. Nephrology. juin 2014;19(6):352-8.

36. Zhang R, Li M, Chouhan KK, Simon EE, Hamm LL, Batuman V. Urine free light chains as a novel biomarker of acute kidney allograft injury. Clin Transplant. nov 2013;27(6):953-60.

37. Seeman T, Vondrak K, Dusek J, Simankova N, Zieg J, Hacek J, et al. Urinary Neutrophil GelatinaseAssociated Lipocalin Does Not Distinguish Acute Rejection from Other Causes of Acute Kidney Injury in Pediatric Renal Transplant Recipients. Clin Lab [Internet]. 2017 [cité 16 juill 2018];63(01/2017). Disponible sur: http://www.clin-lab-publications.com/article/2366

\section{Tables}


Table 1: Patients characteristics at baseline

\begin{tabular}{llc}
\hline Age (years) & Mean (SD) & $51.8(12.5)$ \\
Male gender & $\mathrm{n}(\%)$ & $139(62.3 \%)$ \\
\hline Ethnic origin & $\mathrm{n}(\%)$ & \\
\hline Caucasian/White & & $170(76.2 \%)$ \\
\hline Black & & $41(18.4 \%)$ \\
\hline Asian & & $10(4.5 \%)$ \\
\hline Other & & $2(0.9 \%)$ \\
\hline Weight (kg) & & \\
\hline BMI (kg/m $)$ & Mean (SD) & $70.6(14.0)$ \\
\hline eGFR (mL/min/1.73m $)$ & Mean (SD) & $24.6(4.0)$ \\
\hline Duration of graft at inclusion (months) & Median (IQR) & $3.98(29.40)$ \\
\hline
\end{tabular}

IQR: interquartile range; SD: standard deviation 
Table 2: Patients classification based on main histopathological diagnostics

\begin{tabular}{ccc|}
\hline Iain histopathological diagnostics & Abbreviation & Number of patients \\
\hline Acute cellular rejection & ACR & 18 \\
Acute humoral rejection & AHR & 9 \\
Acute mixed rejection & AMR & 1 \\
Acute tubular necrosis & ATN & 11 \\
BK virus nephropathy & BKVN & 1 \\
Calcineurin inhibitor toxicity & CIT & 5 \\
Chronic cellular rejection & CCR & 1 \\
$\quad$ Glomerulopathy & GP & 20 \\
srstitial fibrosis and tubular atrophy & IFTA & 66 \\
lightly modified renal parenchyma & SMRP & 85 \\
Thrombotic microangiopathy & TMA & 3 \\
$\quad$ Non-assessable & & 2 \\
\hline
\end{tabular}


Table 3 : Grafts classification according to BANFF 2007

\begin{tabular}{l|r|r|r|r|r|}
\hline & Severity grade 0 & Severity grade 1 & Severity grade 2 & Severity grade 3 & Non assessable \\
\hline ali & 164 & 27 & 19 & 11 & 2 \\
\hline & 69 & 75 & 46 & 29 & 4 \\
\hline ial fibrosis & 98 & 83 & 30 & 7 & 5 \\
\hline & 80 & 96 & 30 & 15 & 2 \\
\hline ial infiltrate & 182 & 34 & 4 & 3 & 0 \\
\hline
\end{tabular}


Page 19/24 
Table 4 : Descriptive statistics for urinary biomarkers corrected by urinary creatinine over all patients

\begin{tabular}{|c|c|c|c|c|c|}
\hline & $\mathbf{N}$ & Median & IQR & Min & Max \\
\hline $\operatorname{srin} 2(\mu \mathrm{g} / \mathrm{mmol})$ & 211 & 0.81 & 0.87 & 0.03 & 5.72 \\
\hline$\overline{\operatorname{din}(\mu \mathrm{g} / \mathrm{mmol})}$ & 217 & 4.37 & 8.88 & 0.03 & 105.95 \\
\hline$\overline{\operatorname{cin}(\mu \mathrm{g} / \mathrm{mmol})}$ & 217 & 2.15 & 4.14 & 0.01 & 178.33 \\
\hline $\mathrm{n} \mathrm{C} \mathrm{( \mu g/mmol)}$ & 217 & 4.84 & 18.44 & 0.05 & 2195.65 \\
\hline $\mathrm{\mu g} / \mathrm{mmol})$ & 217 & 0.09 & 0.11 & 0 & 3.35 \\
\hline$(\mu \mathrm{g} / \mathrm{mmol})$ & 215 & 1.2 & 0.82 & 0.14 & 19.64 \\
\hline$\overline{(\mathrm{ng} / \mathrm{mmol})}$ & 217 & 42 & 71.8 & 1.19 & 19696.4 \\
\hline lbumin (mg/mmol) & 217 & 4.72 & 17.4 & 0 & 3535.42 \\
\hline$\overline{\mathrm{JI} / \mathrm{mmol})}$ & 217 & 0.61 & 0.66 & 0 & 27.67 \\
\hline$\overline{\mathrm{n}(\mu \mathrm{g} / \mathrm{mmol})}$ & 214 & 0.08 & 0.06 & 0.02 & 1.02 \\
\hline$\overline{(\mu \mathrm{g} / \mathrm{mmol})}$ & 217 & 5.45 & 11.06 & 0.09 & 2554.17 \\
\hline$\overline{\text { ontin }(\mu \mathrm{g} / \mathrm{mmol})}$ & 217 & 63.23 & 70.78 & 1.61 & 9625 \\
\hline lyxin ( $\mathrm{gg} / \mathrm{mmol})$ & 213 & 2.55 & 1.85 & 0.85 & 60 \\
\hline$\overline{\mathrm{n}(\mu \mathrm{g} / \mathrm{mmol})}$ & 216 & 0.08 & 0.06 & 0.03 & 2.39 \\
\hline$\mu \mathrm{g} / \mathrm{mmol})$ & 217 & 3.47 & 20.58 & 0.53 & 8695.87 \\
\hline 'roteins $(\mathrm{g} / \mathrm{mmol})$ & 213 & 0.02 & 0.04 & 0 & 0.92 \\
\hline
\end{tabular}


Table 5 : Best BM by AUROC in each main histopathological diagnostic group versus control group (SMRP or all other patients) for BM corrected by urinary creatinine

\begin{tabular}{|c|c|c|c|c|}
\hline $\begin{array}{l}\text { Glomerulopathy vs } \\
\text { SMRP }\end{array}$ & ATN vs SMRP & AHR vs SMRP & ACR vs SMRP & IFTA vs SMRP \\
\hline $\begin{array}{l}\text { Microalbuminuria - } 0.98 \\
(0.94 ; 1)\end{array}$ & NGAL - $0.93(0.85 ; 0.99)$ & $\begin{array}{l}\text { BUN - 0.82 } \\
(0.66 ; 0.93)\end{array}$ & $\begin{array}{l}\text { No BM with } \\
\text { AUROC } \geq 0.80\end{array}$ & $\begin{array}{l}\text { No BM with } \\
\text { AUROC } \geq 0.80\end{array}$ \\
\hline $\begin{array}{l}\text { Urinary total protein - } \\
0.97(0.92 ; 1)\end{array}$ & $\begin{array}{l}\text { Urinary cystatin C - } 0.89 \\
(0.75 ; 0.99)\end{array}$ & & & \\
\hline KIM1 - $0.81(0.70 ; 0.90)$ & $\begin{array}{l}\text { Urinary total proteins - } \\
0.89(0.77 ; 0.98)\end{array}$ & & & \\
\hline \multirow{8}{*}{$\begin{array}{l}\text { Clusterin - } 0.80 \\
(0.68 ; 0.88)\end{array}$} & RBP4 - $0.88(0.79 ; 0.96)$ & & & \\
\hline & BUN - $0.88(0.74 ; 0.98)$ & & & \\
\hline & $\begin{array}{l}\text { Serum cystatin } \mathrm{C}-0.88 \\
(0.74 ; 0.97)\end{array}$ & & & \\
\hline & $\begin{array}{l}\text { Serum creatinine }-0.87 \\
(0.69 ; 0.97)\end{array}$ & & & \\
\hline & KIM1 - $0.85(0.72 ; 0.94)$ & & & \\
\hline & $\begin{array}{l}\text { Clusterin - } 0.84 \\
(0.70 ; 0.94)\end{array}$ & & & \\
\hline & $\begin{array}{l}\text { Microalbuminuria - } 0.83 \\
(0.69 ; 0.93)\end{array}$ & & & \\
\hline & MCP1 - $0.82(0.62 ; 0.95)$ & & & \\
\hline $\begin{array}{l}\text { Glomerulopathy vs all } \\
\text { the others }\end{array}$ & ATN vs all the others & $\begin{array}{l}\text { AHR vs all the } \\
\text { others }\end{array}$ & $\begin{array}{l}\text { ACR vs all the } \\
\text { others }\end{array}$ & $\begin{array}{l}\text { IFTA vs all the } \\
\text { others }\end{array}$ \\
\hline $\begin{array}{l}\text { Microalbuminuria - } 0.92 \\
(0.86 ; 0.96)\end{array}$ & NGAL - $0.86(0.75 ; 0.94)$ & $\begin{array}{l}\text { No BM with } \\
\text { AUROC } \geq 0.80\end{array}$ & $\begin{array}{l}\text { No BM with } \\
\text { AUROC } \geq 0.80\end{array}$ & $\begin{array}{l}\text { No BM with } \\
\text { AUROC } \geq 0.80\end{array}$ \\
\hline \multirow[t]{7}{*}{$\begin{array}{l}\text { Urinary total protein - } \\
0.90(0.83 ; 0.96)\end{array}$} & $\begin{array}{l}\text { Urinary cystatin C - } 0.84 \\
(0.67 ; 0.96)\end{array}$ & & & \\
\hline & RBP4 - $0.82(0.75 ; 0.91)$ & & & \\
\hline & $\begin{array}{l}\text { Serum creatinine }-0.81 \\
(0.62 ; 0.84)\end{array}$ & & & \\
\hline & $\begin{array}{l}\text { Serum cystatin C - } 0.81 \\
(0.65 ; 0.92)\end{array}$ & & & \\
\hline & BUN - $0.81(0.64 ; 0.93)$ & & & \\
\hline & MCP1 - $0.80(0.62 ; 0.93)$ & & & \\
\hline & KIM1 - $0.80(0.67 ; 0.91)$ & & & \\
\hline
\end{tabular}


BUN: Blood Urea Nitrogen ; KIM1 : Kidney Injury Molecule-1 ; NGAL: Neutrophil Gelatinase Associated Lipocalin; MCP1 : Monocyte Chemotactic Protein-1; RBP4 : Retinol-Binding Protein-4 ;

ACR : Acute Cellular Rejection; AHR: Acute Humoral Rejection ; ATN: Acute Tubular Necrosis; IFTA: Interstitial Fibrosis and Tubular Atrophy; SMRP: Slightly Modified Renal Parenchyma

AUROC: Area Under ROC curve

Table 6 : Best BM by AUROC to discriminate ATN from acute rejection for biomarkers corrected by urinary creatinine

\begin{tabular}{l|l|l}
\hline ATN vs ACR* & ATN vs AHR* & ATN vs ACR+AHR+AMR \\
\hline eGFR - $0.88(0.72 ; 0.98)$ & Serum creatinine - $0.85(0.63 ; 1)$ & NGAL - $0.85(0.70 ; 0.95)$ \\
\hline NGAL - $0.86(0.70 ; 0.96)$ & eGFR - $0.83(0.61 ; 0.97)$ & eGFR - $0.85(0.67 ; 0.97)$ \\
\hline Serum cystatin C - $0.86(0.65 ; 1)$ & Urinary cystatin C - $0.82(0.55 ; 0.98)$ & Urinary cystatin C - $0.84(0.64 ; 0.97)$ \\
\hline Urinary cystatin C - $0.85(0.67 ; 0.97)$ & NGAL - $0.82(0.56 ; 0.95)$ & Serum cystatin C - $0.84(0.63 ; 0.99)$ \\
\hline Serum creatinine - $0.85(0.63 ; 0.99)$ & RBP4 - $0.82(0.39 ; 0.98)$ & Serum creatinine - $0.83(0.62 ; 0.98)$ \\
\hline MCP1 - 0.82 - 0.61;0.98) & Serum cystatin C - $0.81(0.57 ; 0.98)$ & MCP1 - $0.80(0.47 ; 0.96)$ \\
\hline
\end{tabular}

*The only patient with mixed humoral and cellular rejection was included in ACR and AHR groups.

eGFR: estimated Glomerular Filtration Rate; NGAL: Neutrophil Gelatinase Associated Lipocalin; MCP1 :

Monocyte Chemotactic Protein-1; RBP4 : Retinol-Binding Protein-4 ;

ACR: Acute Cellular Rejection; AHR: Acute Humoral Rejection; AMR: Acute Mixed Rejection; ATN: Acute Tubular Necrosis;

AUROC: Area Under ROC curve

\section{Figures}




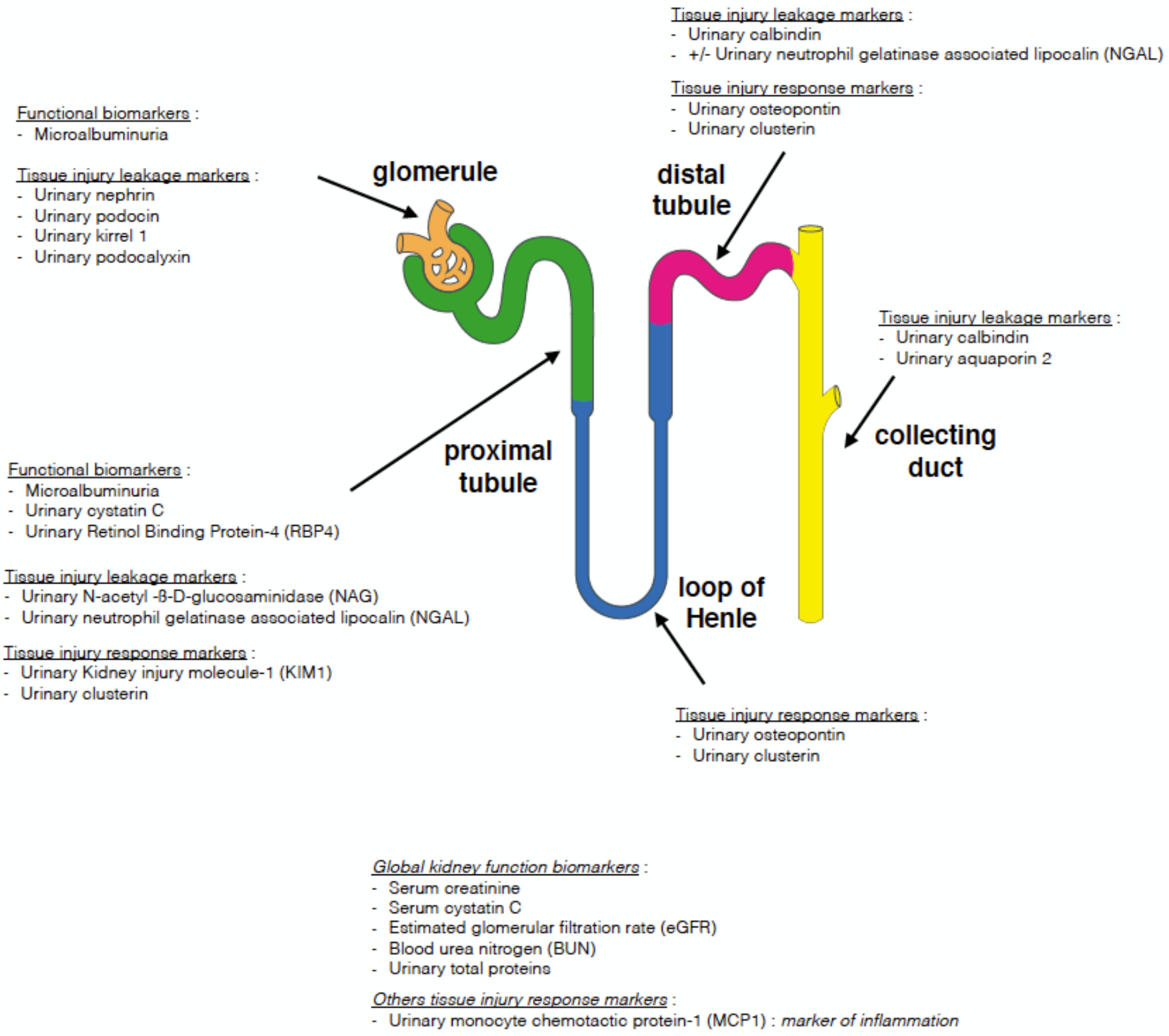

Figure 1

Classification of new biomarkers in different segments of the nephron

\section{Supplementary Files}

This is a list of supplementary files associated with this preprint. Click to download.

- Additionalfile5.doc

- Additionalfile4.doc

- Additionalfile6.doc

- Additionalfile3.doc 
- Additionalfile2.doc

- Additionalfile1.doc 